\title{
Correction to: Lymphovascular invasion, perineural invasion, and tumor budding are prognostic factors for stage I colon cancer recurrence
}

Seijong Kim ${ }^{1}$. Jung Wook Huh ${ }^{1}$. Woo Yong Lee ${ }^{1}$. Seong Hyeon Yun ${ }^{1} \cdot$ Hee Cheol Kim ${ }^{1} \cdot$ Yong Beom Cho ${ }^{1}$. Yoon Ah Park ${ }^{1}$. Jung Kyong Shin ${ }^{1}$

Published online: 4 April 2020

(C) Springer-Verlag GmbH Germany, part of Springer Nature 2020

\section{Correction to: International Journal of Colorectal Disease} https://doi.org/10.1007/s00384-020-03548-4

The name of the author of the original published version of this article was presented incorrectly. The author name "JungWook Huh" should have been presented as "Jung Wook Huh". This has been correctly presented above.

The original article has been corrected.

The online version of the original article can be found at https://doi.org/ $10.1007 / \mathrm{s} 00384-020-03548-4$

Jung Wook Huh

jungwook.huh@gmail.com

1 Department of Surgery, Samsung Medical Center, Sungkyunkwan

University School of Medicine, 81 Irwon-ro, Gangnam-gu,

Seoul 06351, South Korea 